\title{
A Rapid Dilution Technique
}

\author{
By G. D. ANAGNOSTOPOULOS \\ Microbiology Department, Queen Elizabeth College, University of London, \\ Campden Hill, London W.8
}

\section{(Accepted for publication 3 November 1970)}

Viable counts by ordinary dilution procedures and plating is a routine practice in many fields of microbiology, Many attempts have been made to simplify these procedures in terms of both time and material consumed. There are fields of research where sampling at close intervals is essential. In such cases storing of samples, although undesirable because of possible deleterious effects on viability of the bacteria, may become necessary.

Faced with this problem in our growth experiments where very frequent sampling was required, we adopted the technique described below which eliminates the requirement for sterile pipettes and tubes of diluent, thus reducing time spent on preparations of materials and in manipulations. All the apparatus required for this technique is shown in Plate $I$.

The stock diluent is conveniently contained in a Petri dish and is dispensed with a graduated I ml. syringe. The syringe is inserted into the Petri dish through a hole in the plastic lid, and a diluent volume of $0.9 \mathrm{ml}$. is transferred into a glass dish previously sterilized by burning alcohol. No change of syringe has been found necessary during platings over 7 to $8 \mathrm{~h}$. The inoculum is measured by means of a pipette delivering $50 \mathrm{drops} / \mathrm{ml}$. of the diluent used. Five drops of inoculum mixed with the diluent give a I/Io dilution. Alternate use of two pipettes saves waiting time for cooling. The main feature is that the diluent and inoculum are placed in a small glass dish $4 \times 4 \times \mathrm{I} \cdot 5 \mathrm{~cm}$. with a hemispheric cavity $\mathrm{I} \cdot 5 \mathrm{~cm}$. wide and $\mathrm{I} \cdot 2 \mathrm{~cm}$. deep (Baird and Tatlock) and mixed by means of a magnetic stirrer $6 \mathrm{~mm}$. long and about $0.5 \mathrm{~mm}$. thick sealed in a piece of capillary tubing (Voss Instruments, Faraday Works, Maldon, Essex). In the meantime the inoculating pipette is rinsed in alcohol and ignited and the diluent for the next dilution is placed in another sterile glass dish.

After plating the appropriate dilutions of culture, all glass dishes are vacuumemptied into a conical flask connected to a vaccum line. Plugging the nozzle into a rubber stopper is a quick method of sealing the vacuum line. The dishes are then wetted with a piece of cotton wool soaked in alcohol and the residual alcohol ignited. The magnets, similarly sterilized, are replaced in the dishes which can then be stored under a large Petri dish lid. The whole procedure of making a dilution up to $10^{-6}$, plating, cleaning and sterilization takes no longer than 6 min.

No special contamination problems have been experienced over a period of several months. No aerosol could be detected with agar plates placed over the glass dishes during the magnetic mixing, but application of this technique to pathogenic organisms would require additional precautions, e.g. use of a fan cabinet and covers for the glasses. We find that this technique is considerably faster and more convenient than other 
dilution procedures and makes possible sampling and plating at intervals as close as 6 to $8 \mathrm{~min}$. It is also economical in terms of materials, technical assistance and effort.

\section{EXPLANATION OF PLATE}

An illustration of equipment for the dilution technique. 
Journal of General Microbiology, Vol. 64, No. 2

Plate I

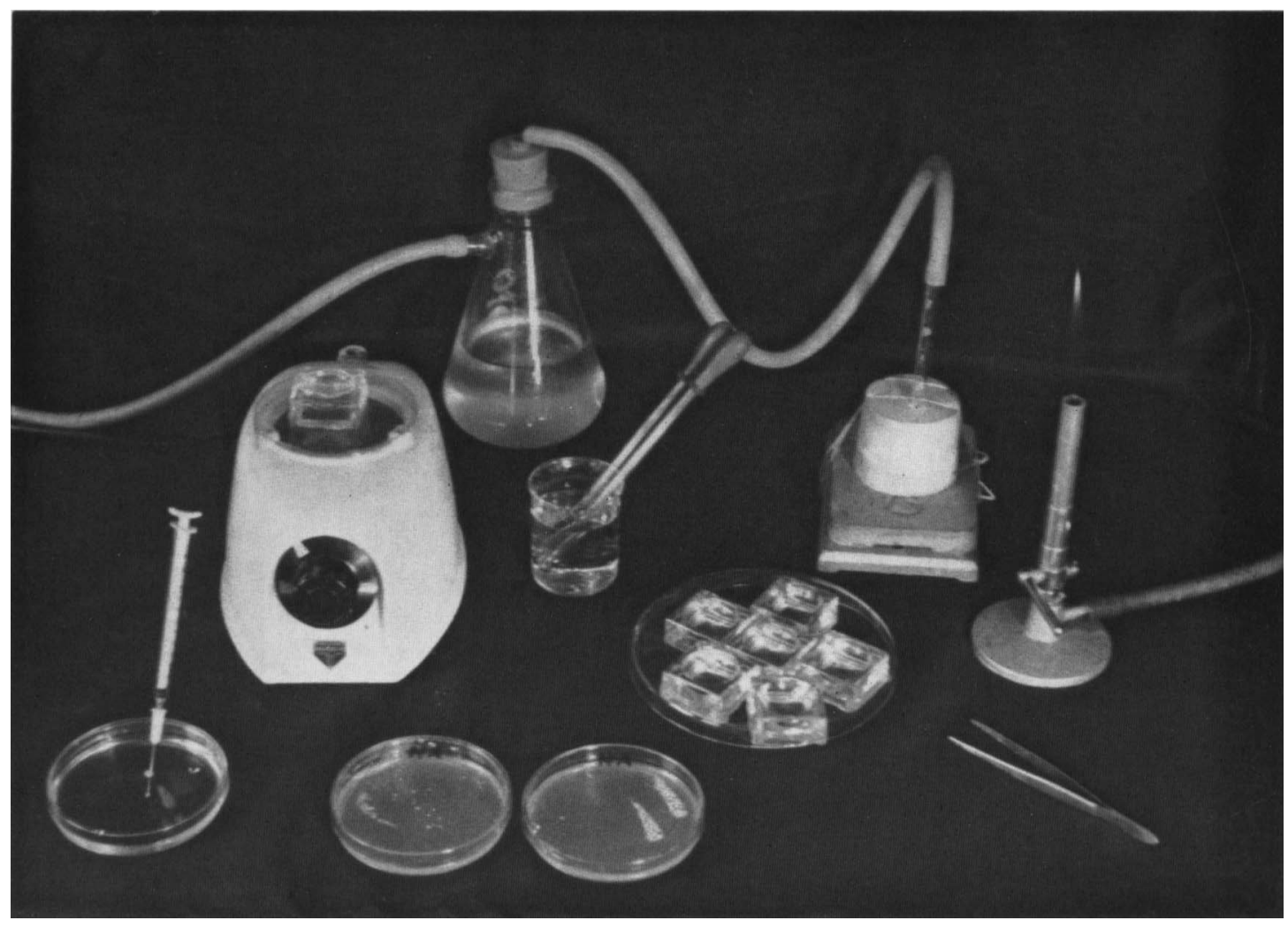

